\title{
On the theory of equilibrium magnetoelastic domain structure in easy-plane antiferromagnet
}

\author{
E. V. Gomonaj ${ }^{1}$ and V. M. Loktev ${ }^{1,2}$ \\ ${ }^{1}$ National Technical University of Ukraine KPI, 37, Ave. Peremogy, Kiev, 252056, Ukraine \\ ${ }^{2}$ Bogolyubov' Institute for Theoretical Physics, National Academy of Sciences of Ukraine, 14-b, \\ Metrologichna str., Kiev, 252143, Ukraine \\ E-mail: malyshen@ukrpack.net \\ vloktev@bitp.kiev.ua \\ Received March 5, 1999
}

\begin{abstract}
Macroscopic magnetoelastic domain structure of the defectless layered antiferromagnet of $\mathrm{CoCl}_{2}$-type with the «easy-plane» magnetic anisotropy is studied theoretically in the framework of phenomenological approach. In assumption of mobile domain walls, the finite-size effects are shown to result in the formation of a stable domain structure that changes reversibly under the action of the external magnetic field and can be treated as equilibrium. It is found that in antiferromagnets, where (in contrast to ferromagnets) long-range forces of magnetic origin are absent, the domain structure and its collective behavior are governed by elasticity. Field dependence of a domain structure, magnetostriction and low-frequency AFMR of poly- and monodomain samples are calculated, the external magnetic field being directed perpendicular to the main symmetry axis of the crystal. The results obtained are in qualitative agreement with the available experimental data.
\end{abstract}

PACS: 75.50.Ee, 75.60.Ch

\section{Introduction}

The origin of equilibrium domain structure (DS) in antiferromagnetic (AFM) insulators is studied for a long time [1,2], but the question is still obscure, despite a well developed theory for the close vicinity of the magnetic 1-st order phase transitions (in particular, for the field-induced spinflop transitions in the easy-axis antiferromagnets (see the review [3] and recent paper [4] where this theory has been generalized for the case of the hexagonal AFM with the easy-plane magnetic anisotropy)). The DS of the pure antiferromagnets is usually treated as the result of structural imperfections (such as dislocations, twins, impurities, etc.) that cause the so-called sprout AFM domains (including $180^{\circ}$ domains). Sometimes, the origin of the DS is attributed to the entropy factor, which decreases the free energy of the sample in the vicinity of the critical temperature in the case of spatially inhomogeneous ordering.
Both mentioned and some other possibilities were analyzed in a recent paper [5] where numerous experimental evidences of the equilibrium (almost insensitive to growth conditions) domain structure in dihaloids of transition metals $\mathrm{MX}_{2}(\mathrm{M}=\mathrm{Mn}$, $\mathrm{Co}, \mathrm{Ni} ; \mathrm{X}=\mathrm{Cl}, \mathrm{Br}$ ) were given. According to observations, the domain structure changes almost reversibly under the action of the external magnetic field; it gradually disappears when the field is switched on and recovers after it is switched off. Such a behavior evidently points to the equilibrium nature of the DS observed in these compounds* .

The authors [5] also suggested that magnetoelastic interactions play a dominant role in formation of the equilibrium DS in the layered easy-plane antiferromagnets of $\mathrm{CoCl}_{2}$-type. However, the only condition pointed out is not sufficient and there is an additional requirement necessary for DS formation, namely, the account of the sample surface. The finite size effects bring about the appearance of the DS during ferromagnetic and ferroelastic phase

* We cannot exclude another possibility, when, for example, due to the high defect concentration, the internal stresses govern the local equilibrium orientation of AFM vector. After the magnetic field is removed, this vector reverts to the initial state or to the nearest easy direction. On the other hand, observations [5] point to the regular DS rather than to the stochastic. 
transitions as well as the magneto-elastic interactions.

These effects should play an important role in the AFM, and particularly, in dihaloids of transition metals, where the antiferromagnetic domains bear a magnetostrictive character and are of rather small size. In fact, the width of the antiferromagnetic domain wall can be evaluated as $\delta_{0} \sim a\left(2 H_{E} / H_{A}\right)^{1 / 2} \sim a\left(2 H_{E} / H_{s f}\right) \sim 15-20 a$, where $a$ is interatomic distance, $H_{E}, H_{A}$ and $H_{s f}$ are the exchange field, the interplane anisotropy field and the spin-flop field, correspondingly (for $\mathrm{CoCl}_{2}$ $H_{s f}=2 \mathrm{kOe}[6]$ and spin-flip field $2 H_{E}=32 \mathrm{kOe}$ [7]). Small thickness and low energy of the domain walls $\left(2 \cdot 10^{-4} \mathrm{~mJ} / \mathrm{m}^{2}\right.$, compared with the typical value $4 \mathrm{~mJ} / \mathrm{m}^{2}$ for a ferromagnet) give grounds for expecting the small size of AFM domains and assume that the surface properties of the sample do strongly depend upon the average strain.

It should be mentioned that an equilibrium DS is observed in the martensites in the course of a thermoelastic phase transition. It arises from the condition of strain compatibility of martensitic and austenitic phases. The peculiar feature of this structure is that the domain (twin) size is very small $(\sim 1000 \AA$, see [8]) and compatibility conditions relate only with the average-strain.

The present paper is aimed at the theoretical investigation of the macroscopic magnetoelastic domain structure of the defectless easy-plane layered AFM of $\mathrm{CoCl}_{2}$-type with the account of finite-size (surface) effects. In the framework of the phenomenological model, we calculate the domain structure, sample magnetostriction and AFMR frequency in the presence of an external magnetic field directed perpendicular to the main symmetry axis of the crystal.

\section{Model}

We consider a thin plate of a layered easy-plane rhombohedral antiferromagnet of $\mathrm{CoCl}_{2}$-type. The crystal symmetry group is $D_{3 d}^{5}$. The plate is oriented perpendicular to the 3-rd order crystal axis, labeled as $z, x$-axis is chosen along the 2-nd order in-plane symmetry axis. Below the Néel temperature, the magnetic structure of the crystal can be described with two orthogonal dimensionless vectors: ferromagnetic, m, and antiferromagnetic, 1,

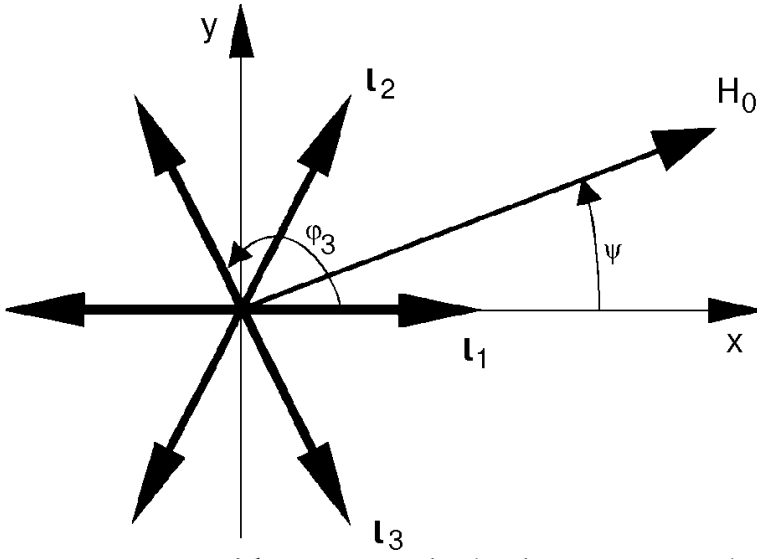

Fig. 1. Orientation of $\mathbf{l}$ vectors inside the domains, $\mathbf{H}_{0}$ is the external magnetic field.

$\mathbf{m}^{2}+\mathbf{l}^{2}=1$. In the absence of external fields $\mathbf{m}=0$ and $\mathbf{1}$ has 3 equivalent orientations ${ }^{*}$ in the basis plane (directed along three 2-nd order symmetry axes). In the in-plane external magnetic field, the magnetic structure is described by two parameters: the modulus $m$ of the ferromagnetic vector, and the angle $\varphi$ between the $\mathbf{l}$ and $x$-axes, neglecting small deflections of the magnetic vectors from the basis plane (see Fig. 1). The bulk free energy of the crystal in this case can be written in the simplest form (see, e. g., [9]):

$$
\begin{gathered}
F_{\mathrm{vol}}=\int d v\left\{2 J M_{0}^{2} \mathbf{m}^{2}+2 \beta^{(2)} M_{0}^{2} m_{z}^{2}-\right. \\
-\frac{2}{3} \beta_{\perp}^{(6)} M_{0}^{2}\left(1-m^{2}\right)^{3} \cos 6 \varphi+4 M_{0}^{2}\left[\lambda_{m e}^{(l)}\left(1-m^{2}\right)+\right. \\
\left.+\lambda_{m e}^{(m)} m^{2}\right]\left[\left(u_{x x}-u_{y y}\right) \cos 2 \varphi+2 u_{x y} \sin 2 \varphi\right]+
\end{gathered}
$$$$
\left.+\frac{1}{2} c_{66}\left[\left(u_{x x}-u_{y y}\right)^{2}+4 u_{x y}^{2}\right]-2 M_{0} H_{0} m \sin (\varphi-\psi)\right\},
$$

where the constant $\mathrm{J}$ describes the interplanar AFM exchange; $\beta^{(2)}, \beta_{\perp}^{(6)}$ are the effective anisotropy constants; $2 M_{0}$ is the saturation magnetization; $\lambda_{m e}$ are the magnetostrictive constants; $u_{i k}(i, k=x, y)$ are the strain tensor components; $c_{66}$ is the elastic modulus, principal for the case under consideration, and the external magnetic field is defined as $H_{0}=\left|\mathbf{H}_{0}\right|, \tan \psi=H_{0 y} / H_{0 x}$. In the expression (1) we have omitted the isotropic part of strain tensor, $u_{x x}+u_{y y}$, qualitatively insignificant for the pre-

* We don't distinguish between the states with $\mathbf{1}$ and $-\mathbf{1}$. Besides, these three directions are usually provided by hexagonal anisotropy, as it was suggested in [4]; but in rhombohedral AFM this anisotropy can be also caused by the difference in strain components $u_{x z}, u_{y z}$ that results in $60^{\circ}$ in-plane anisotropy for 1 vector. In what follows, however, we shall suppose that there is an in-plane magnetic anisotropy which effectively includes both these factors. So, corresponding strain components will be omitted for simplicity. 
sent problem. The effective magnetostrictive constants $\lambda_{m e}^{(l)}$ and $\lambda_{m e}^{(m)}$ originate from the relativistic (dipole-dipole or spin-orbit) interactions and, as was shown in [10], can essentially depend upon the concrete electronic and crystal structure of a compound.

Herein we consider the model in which the surface effects are accounted through the surface tension. The shape-dependent part of the surface energy for the simplest case of disk with radius $R$ and thickness $h$ is given by the expression

$$
F_{\text {surf }}=\frac{\pi R h}{2} \sigma_{\text {surf }}\left[\left\langle u_{x x}-u_{y y}\right\rangle^{2}+4\left\langle u_{x y}\right\rangle^{2}\right]
$$

where $\sigma_{\text {surf }}$ is the surface tension coefficient for the (100) and (010) faces, notion $\langle\ldots\rangle$ means the averaging over the sample volume:

$$
\left\langle u_{i j}\right\rangle=\frac{1}{\pi R^{2} h} \int u_{i j} d v
$$

and we have neglected the contribution from the disc sides (001) (which is appropriate if $h<<R$ ). In the expressions (1), (2) we have neglected the magnetostatic energy which contribution, as will be shown below, is much smaller than that from the surface energy.

For small external fields, $H_{0} \ll J M_{0}$, the ferromagnetic moment $m<<1$ can easily be excluded from (1). So, neglecting $\beta^{(2)}<<J$,

$$
\begin{gathered}
F_{\mathrm{vol}}=\int d v\left\{-\frac{2}{3} \beta_{\perp}^{(6)} M_{0}^{2} \cos 6 \varphi-\frac{H_{0}^{2}}{2 J} \sin ^{2}(\varphi-\psi)+\right. \\
+4 M_{0}^{2} \lambda_{m e}^{(l)}\left[\left(u_{x x}-u_{y y}\right) \cos 2 \varphi+2 u_{x y} \sin 2 \varphi\right]+ \\
\left.+\frac{1}{2} c_{66}\left[\left(u_{x x}-u_{y y}\right)^{2}+4 u_{x y}^{2}\right]\right\} .
\end{gathered}
$$

The local orientation of the vector 1 can then be found by minimization of the functional

$$
F=F_{\text {vol }}+F_{\text {surf }}
$$

with respect to $\varphi(\mathbf{r}), u(\mathbf{r})$ functions. The corresponding integral equations have the form:

$$
\begin{aligned}
& \left\{\begin{array}{l}
u_{x x}-u_{y y}=-\frac{4 M_{0}^{2} \lambda_{m e}^{(l)}}{c_{66}} \cos 2 \varphi+\frac{4 M_{0}^{2} \lambda_{m e}^{(l)} \sigma_{\text {surf }}}{c_{66}\left(c_{66} R+\sigma_{\text {surf }}\right)}\langle\cos 2 \varphi\rangle \\
2 u_{x y}=-\frac{4 M_{0}^{2} \lambda_{m e}^{(l)}}{c_{66}} \sin 2 \varphi+\frac{4 M_{0}^{2} \lambda_{m e}^{(l)} \sigma_{\text {surf }}}{c_{66}\left(c_{66} R+\sigma_{\text {surf }}\right)}\langle\sin 2 \varphi\rangle
\end{array}\right. \\
& \frac{1}{3} H_{s f}^{2} \sin 6 \varphi=\sin 2 \varphi\left[H_{0}^{2} \cos 2 \psi+2 H_{M D}^{2}\langle\cos 2 \varphi\rangle\right]-\cos 2 \varphi\left[H_{0}^{2} \sin 2 \psi+2 H_{M D}^{2}\langle\sin 2 \varphi\rangle\right] .
\end{aligned}
$$

Here we have introduced the characteristic fields convenient for further calculations: $H_{s f}==2 M_{0} \sqrt{6 \beta_{\perp}^{(6)} J}$ - spin-flop field, and $H_{M D}=4 M_{0}^{2} \lambda_{m e}^{(l)}\left[\left(2 J \sigma_{\text {surf }}\right) / c_{66}\left(c_{66} R+\sigma_{\text {surf }}\right)\right]^{1 / 2}-$ the field of monodomenization.

Equation (7) evidently shows that the surface produces the same effect as an external magnetic field, the effective internal field being defined as

$$
\begin{gathered}
H_{\mathrm{eff}}^{2}=\sqrt{H_{0}^{4}+4 H_{M D}^{4}\left(\langle\cos 2 \varphi\rangle^{2}+\langle\sin 2 \varphi\rangle^{2}\right)+2 H_{0}^{2} H_{M D}^{2}\langle\cos 2(\varphi-\psi)\rangle^{2}} \\
\tan 2 \psi_{\mathrm{eff}}=\frac{H_{0}^{2} \sin 2 \psi+2 H_{M D}^{2}\langle\sin 2 \varphi\rangle}{H_{0}^{2} \cos 2 \psi+2 H_{M D}^{2}\langle\cos 2 \varphi\rangle} .
\end{gathered}
$$

Thus, it can be stressed that in the case of AFM it is the elastic strain that plays the role similar to the magneto-dipole interaction in ferromagnets.

Note, that for infinite sample $(R \rightarrow \infty) H_{M D} \rightarrow 0$ and effective field identically coincides with the external field $\mathbf{H}_{0}$; for such a situation $\psi_{\text {eff }} \rightarrow \psi$. o.

Equation (7) has different solutions depending on the physical situation considered below.

\subsection{Mobile domain walls}

In this case the average strain can follow the changes caused by an external magnetic field. Up to a certain field value, $H_{0}=H_{1 c}$, specified below, the effect of the magnetic field is compensated by the average strains, so that the effective field inside the sample $\mathbf{H}_{\mathrm{eff}}=0^{*}$. Equation (7) has three non- 
trivial solutions $\varphi_{1}=0, \varphi_{2,3}= \pm 2 \pi / 3$, corresponding to 3 equivalent equilibrium orientations of the 1 vector, i.e., to three magnetoelastic domains (as was already pointed out before). Evidently, each of them is distorted orthorhombically [in correspondence with [11,12], see formulas (6)]. Such a distortion for easy-plane AFM was observed in [13]. Moreover, magnetoelasticity proves to be a crucial factor for the existence of an equilibrium DS.

In neglecting the domain wall energy, one can find the relative volume $\xi_{j}(j=1,2,3)$ of each domain from the following equations, obtained from (8):

$$
\begin{aligned}
& H_{0}^{2} \cos 2 \psi+2 H_{M D}^{2}\langle\cos 2 \varphi\rangle=0 \\
& H_{0}^{2} \sin 2 \psi+2 H_{M D}^{2}\langle\sin 2 \varphi\rangle=0
\end{aligned}
$$

we take it into account that:

$$
\langle\cos 2 \varphi\rangle=\sum_{j} \xi_{j} \cos 2 \varphi_{j}, \sum_{j} \xi_{j}=1 .
$$

The ultimate expression for $\xi_{j}$ is

$$
\xi_{j}=\frac{1}{3}\left[1-\frac{H_{0}^{2}}{H_{M D}^{2}} \cos 2\left(\varphi_{j}-\psi\right)\right], j=1,2,3 .
$$

Note, that in this case $\xi_{j}$ are the thermodynamic variables as well as strain components and $\varphi$; the equality of the chemical potentials (free energy densities) of different «phases» (domains) is satisfied automatically.

According to (12), the volume fraction of the domains depends upon the value of the external magnetic field $H_{0}$. At zero field, $H_{0}=0$, all three types are equally distributed, so that the symmetry of the sample does not change after the transition into the polydomain antiferromagnetic state. In nonzero field the fraction of the most energetically «unfavorable» domain ( 1 vector lies closely to the direction of the magnetic field, say, domain 1 for $0<\psi<\pi / 6$, see Fig. 1) diminishes. At $H_{0}=$ $=H_{1 c}=H_{M D} /(\cos 2 \psi)^{1 / 2}$ the domains of the 1 -st type disappear. Further behavior of the system at $H_{0}>H_{1 c}$ can be found out from the equations (7), (10) and (11) with $j=2,3$. In this case the internal effective field is no longer zero, but it is directed along $x$-axis $\left(\psi_{\text {eff }}=0\right)^{* *}$, so, $\varphi_{2}=-\varphi_{3}=\varphi$, and

$$
\begin{gathered}
\cos 2 \varphi=\frac{3 H_{M D}^{2}}{4 H_{s f}^{2}}- \\
-\frac{1}{2}\left[\left(1+\frac{3 H_{M D}^{2}}{2 H_{s f}^{2}}\right)^{2}+3 \frac{H_{0}^{2}-H_{1 c}^{2}}{H_{s f}^{2}} \cos 2 \psi\right]^{1 / 2}, \\
\xi_{2,3}=\frac{1}{2}\left[1 \mp \frac{H_{0}^{2} \sin 2 \psi}{H_{M D}^{2} \sin 2 \varphi}\right]
\end{gathered}
$$

In other words, in the two-domain structure the $\mathbf{l}$ vectors inside the domains start to rotate and simultaneously the fractions of the different domains change. The process of monodomenization is completed at some critical field $H_{0}=H_{c}$ which can be found from equation (13) along with the condition $\xi_{2}=0$ or in other words,

$$
H_{c}^{2} \sin 2 \psi=H_{M D}^{2} \sin 2 \varphi .
$$

For the case $\psi=0$ both domains 2 and 3 disappear simultaneously at

$$
H_{0}=H_{2 c} \equiv \sqrt{H_{s f}^{2}+2 H_{M D}^{2}},
$$

when all the $\mathbf{l}$ vectors achieve the direction perpendicular to the external field. Effective field (16) of monodomenization is defined both by the magnetic anisotropy (due to $H_{s f}$ ) and by the surface effect (due to $H_{M D}$ ). For the symmetric case $\psi=\pi / 6$ monodomenization is completed at $H_{0}=H_{1 c}$ when domains 1 and 2 disappear and $\mathbf{l}$ vector in the third domain is aligned perpendicular to the external field. For the general case, $0<\psi<\pi / 6$, critical field $H_{1 c}<H_{c}<H_{2 c}$; after the process of monodomenization is finished, further change of $\varphi$ angle can be calculated from the equation

$$
\frac{1}{3} H_{s f}^{2} \sin 6 \varphi=H_{0}^{2} \sin 2(\varphi-\psi) .
$$

The considered model gives rise to thermodynamically equilibrium domain structure at any magnetic field value. Really, the difference in free energy of the polydomain and monodomain state calculated from (1), (2) at the same external field value,

* The similar equality holds true for DS corresponding to the so-called intermediate state that exists in the vicinity of the 1-st order spin-reorientation phase transition in the easy-axis AFM [3].

** It is easy to check that another solution of (7), (8) with $\psi_{\mathrm{eff}}=\pi / 2$ is energetically unfavorable. 


$$
\begin{gathered}
F_{\text {poly }}-F_{\text {mono }}=-\frac{V}{2 J}\left[H_{M D}^{2}+\frac{1}{2} H_{0}^{2} \cos 2(\varphi-\psi)+\right. \\
\left.+\frac{1}{18} H_{s f}^{2}(1-\cos 6 \varphi)\right] \leq 0
\end{gathered}
$$

is nonpositive, which makes the polydomain state thermodynamically preferable. So, in the model proposed the behavior of the DS in the external magnetic field is absolutely reversible.

\subsection{Immobile domain walls}

The domain walls cannot move freely, so, the ratio of the domains is fixed and only the rotational processes take place inside the domains. Orientation of 1 vectors can be calculated from equations (7), (11) with the given $\xi_{j}$ values which are defined by technological factors. For small external field the equation (7) has 3 solutions corresponding to different domains. Monodomenization of the sample is completed when all the $\mathbf{l}$ vectors are aligned perpendicular to the external field direction.

For illustration let us consider the symmetrical case $\psi=0$. If initially the domains have been produced by stray field at random, then $\xi_{1}=\xi_{2}=$ $=\xi_{3}=1 / 3$ and

$$
\begin{gathered}
\varphi_{1}=0, \varphi_{2}=-\varphi_{3}=\varphi \\
\cos 2 \varphi=\frac{H_{M D}^{2}}{2 H_{s f}^{2}}-\frac{1}{2}\left[\left(1+\frac{H_{M D}^{2}}{H_{s f}^{2}}\right)^{2}+3 \frac{H_{0}^{2}}{H_{s f}^{2}}\right]^{1 / 2} .
\end{gathered}
$$

The field of monodomenization $H_{c}^{\mathrm{immob}}=$ $=\left(H_{s f}^{2}+{ }^{2} / 3 H_{M D}^{2}\right)^{1 / 2}$ in this case is smaller than the corresponding value for the case of mobile domain walls [ $H_{c}^{\text {immob }}<H_{2 c}$, compare with formula (16)]. For arbitrary $\psi$ the value of monodomenization field can be much greater.

After the magnetic field is removed, such a domain structure will not restored, at least, in principal, because 1 vectors will tend to lie along the nearest easy axis, which for general field orientation is the only one. So, once cycled in the magnetic field, the sample becomes monodomain and the behavior of the DS in this case is absolutely irreversible.

In the real experiments, the behavior of the DS in antiferromagnets of $\mathrm{CoCl}_{2}$-type are partly irreversible, so, we can assume some intermediate case when most of the domain walls are mobile but some of them are pinned by the defects or different imperfections of a crystal and contribute to the certain irreversibility mentioned and observed.

\section{Magnetostriction}

In the experiments $[5,7]$ the magnetic field was arbitrarily oriented in the easy plane of the crystal, and magnetostriction was measured along and perpendicular to the field direction. Corresponding macroscopic elongations, $(\Delta l / l)_{\|}$and $(\Delta l / \ell)_{\perp}$ can be calculated according to the general formula

$$
(\Delta \mathcal{L} / \mathcal{L})_{\mathbf{n}}=\sum n_{i}<u_{i k}>n_{k}
$$

where $\mathbf{n}$ is a unit vector in the direction of measurement, $\left\langle u_{i k}>\right.$ is the averaged strain tensor. Substituting (6) into (20) and neglecting of isomorphous strain $\left(u_{x x}+u_{y y}\right)$ one readily obtains:

$$
\left(\frac{\Delta l}{l}\right)_{\|}=-\left(\frac{\Delta l}{l}\right)_{\perp}=
$$

$$
=-\frac{4 M_{0}^{2} R\left[\lambda_{m e}^{(l)}+\left(\lambda_{m e}^{(m)}-\lambda_{m e}^{(l)}\right)\left(\frac{H_{0}}{2 H_{E}}\right)^{2}\right]}{c_{66} R+\sigma_{\text {surf }}}\langle\cos 2(\varphi-\psi)\rangle,
$$

where $H_{E}=J M_{0}$, is the exchange field. In the formula (21) we have taken into account the dependence $m\left(H_{0}\right) \approx H_{0} / 2 H_{E}$, which is significant at $H_{0} \leq 2 H_{E}$.

Field dependence of elongation $(\Delta l / l)_{\|}$calculated from (21) for $\mathrm{CoCl}_{2}$ with $\psi=0, H_{s f}=2 \mathrm{kOe}$; $H_{M D}=3.3 \mathrm{kOe}, \quad 2 H_{E}=32 \mathrm{kOe}, \quad 4 M_{0}^{2} \lambda_{m e}^{(l)}=$ $=-4 M_{0}^{2} \lambda_{m e}^{(m)}=36 \quad \mathrm{MPa}, \quad c_{66}=34.7 \mathrm{GPa}$ [7] is shown in Fig. 2. We have considered two cases: mobile (solid curve) and immobile (dash curve) domain waIls. Figure 3 shows the same dependences vs squared magnetic field, $H_{0}^{2}$; the points correspond to experimental data [7]. The difference between two theoretical curves is significant at low field value. In the case of mobile domain walls the theoretical dependence (solid curve) is in good agreement with the experimental data. The $H_{M D}$ value was taken to fit the experimental slope $(\Delta \mathcal{l} / \mathcal{l})_{\perp}$ vs $H_{0}^{2}$ at $H_{0}<3 \mathrm{kOe}$.

The behavior of magnetostriction as seen from formula (21) and Fig. 2 is governed by two processes. At small field, $H_{0} \leq H_{c} \ll H_{E}$, magnetostriction of the sample is changed due to the process of monodomenization that influences the average $\cos 2(\varphi-\psi)$ value (increasing section of the curve in Fig. 2). After this process is finished, variation of monodomain magnetostriction is defined only by an increase of magnetization in the external magnetic field (decreasing section of the 


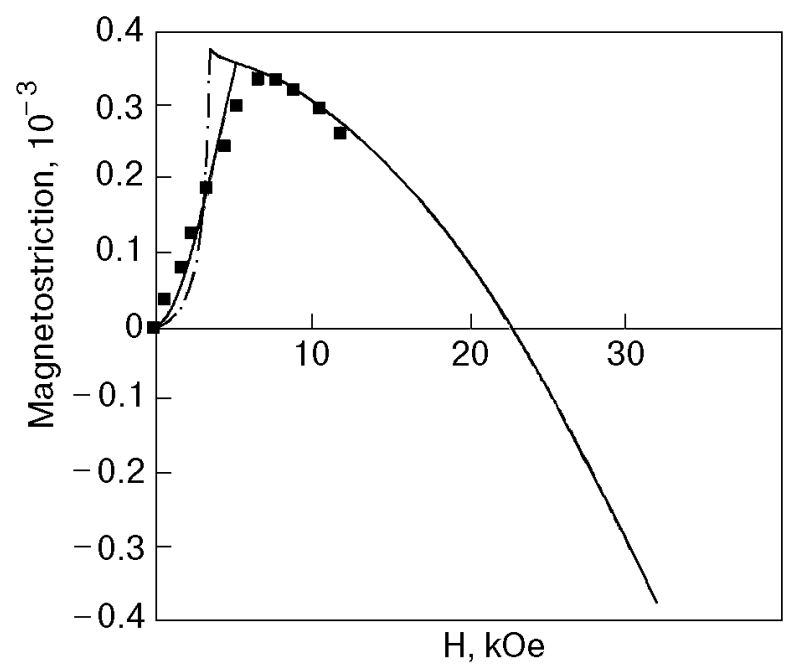

Fig. 2. Field dependence of magnetostriction of polydomain crystal: solid line - theoretical, mobile domain walls; dash line - theoretical, immobile domain walls; points - experimental [7].

curve in Fig. 2). It is seen from (21) that in the monodomain state the slope $(\Delta l / \zeta)_{\perp}$ vs $H_{0}^{2}$ depends upon the difference $\lambda_{m e}^{(m)}-\lambda_{m e}^{(l)}$ only, that must be considered as a phenomenological parameter.

\section{AFMR spectra}

Experimentally measured field dependence of the low-frequency AFMR [7] shows that below $5 \mathrm{kOe}$ the resonance frequency is approximately two times lower than the value extrapolated from measurements at higher frequency. In the interval $\mathrm{H}_{0} \sim 5^{-}$ $7 \mathrm{kOe}$ field dependence of AFMR is practically vertical and starting from $7 \mathrm{kOe}$ it turns out to be in agreement with high-frequency measurements. The characteristic field value coincides with the value of monodomenization field $H_{c}$ observed in magnetostriction experiments.

The observed peculiarity in the AFM spectra can be interpreted in the framework of the equilibrium magnetoelastic domain model developed above. Indeed, for the infinite samples the low AFMR frequency is contributed by the magnetic anisotropy

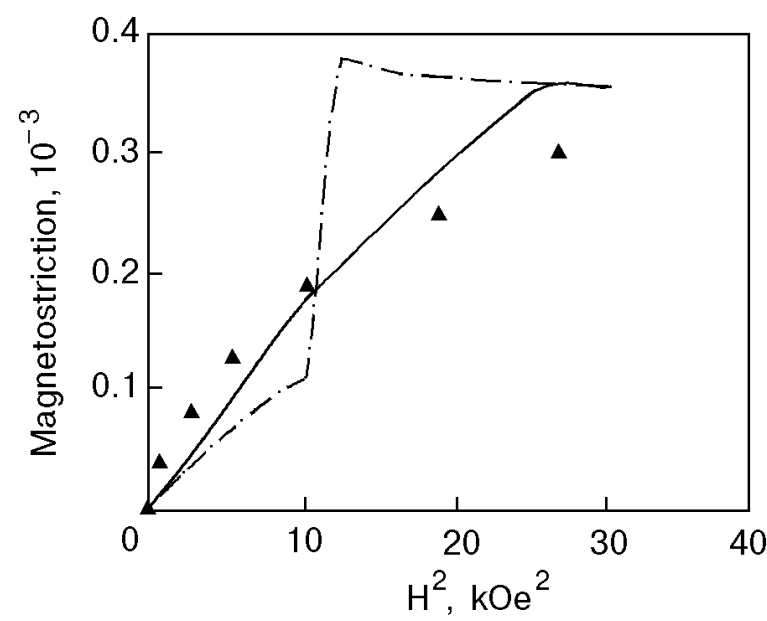

Fig. 3. Magnetostriction vs squared magnetic field in polydomain crystal: solid line - theoretical, mobile domain walls; dash line - theoretical, immobile domain walls; points - experimental [7].

field and magnetoelastic field as well, due to the fact, that the crystal lattice is «frozen». On the other hand, it was shown by Gann and Zhukov [14] that for small samples the lattice relaxes together with the antiferromagnetic vectors, and then, the resonance frequency is defined mainly by the local anisotropy field.

The similar effect of «unfreezing» of crystal lattice can be achieved in the AFM with the magnetoelastic DS, if the domain size is quite small. To catch the effect, let us consider the in-plane oscillations of the magnetic moments together with acoustic waves in the polydomain sample with mobile domain walls. Low-frequency AFMR can be found on the basis of Lagrangian formalism with a $\mathrm{La}^{-}$ grangian taken in a standard form [15]:

$$
L=\int d v\left(\frac{\dot{\varphi}^{2}}{2 J g^{2}}+\frac{1}{2} \rho \dot{\mathbf{u}}^{2}\right)-F_{\mathrm{vol}},
$$

where $g$ is gyromagnetic ratio, $\rho$ is a crystal density, $\mathbf{u}$ is a displacement vector, $F_{\text {vol }}$ is given by formula (4). Corresponding Euler-Lagrange equations have the form:

$$
\left\{\begin{array}{l}
\ddot{\varphi}-\frac{g^{2}}{2} H_{0}^{2} \sin 2(\varphi-\psi)+\frac{g^{2}}{3} H_{s f}^{2} \sin 6 \varphi-16 g^{2} \lambda_{m e}^{(l)} J M_{0}^{2}\left[\left(u_{x x}-u_{y y}\right) \sin 2 \varphi-2 u_{x y} \cos 2 \varphi\right]=0 \\
\ddot{u}_{x}-s^{2}\left[\frac{\partial^{2}}{\partial x^{2}}+\frac{\partial^{2}}{\partial y^{2}}\right] u_{x}-\frac{4 \lambda_{m e}^{(l)} M_{0}^{2}}{\rho}\left[\frac{\partial \cos 2 \varphi}{\partial x}+\frac{\partial \sin 2 \varphi}{\partial y}\right]=0 \\
\ddot{u}_{y}-s^{2}\left[\frac{\partial^{2}}{\partial x^{2}}+\frac{\partial^{2}}{\partial y^{2}}\right] u_{y}-\frac{4 \lambda_{m e}^{(l)} M_{0}^{2}}{\rho}\left[\frac{\partial \sin 2 \varphi}{\partial x}-\frac{\partial \cos 2 \varphi}{\partial y}\right]=0,
\end{array}\right.
$$


where $s=\left(c_{66} / \rho\right)^{1 / 2}$ is the in-plane sound velocity and equilibrium $\varphi$ value depends upon the $x$ and $y$ coordinates. Equations (23) describe the perturbations over inhomogeneous (polydomain) state of the sample.

The low-frequency branch of AFMR for $H_{0}<H_{1 c}$ can be then calculated from the following equation

$$
\omega^{2}=g^{2}\left\{H_{s f}^{2}+H_{M E}^{2}\left[1-\int \frac{\mathbf{k}^{2}\left(\left|a_{\mathbf{k}}\right|^{2}+\left|b_{\mathbf{k}}\right|^{2}\right)}{\mathbf{k}^{2}-\left(\omega^{2} / s^{2}\right)} d \mathbf{k}\right]\right\},
$$

where $H_{M E}=8 M_{0}^{2} \lambda_{m e}^{(l)} \sqrt{J / c_{66}}$ is a magnetostriction field; $a_{\mathbf{k}}, b_{\mathbf{k}}$ are the Fourrier components of the functions $\sin 2 \varphi(\mathbf{r})$ and $\cos 2 \varphi(\mathbf{r})$, correspondingly, $\int\left(\left|a_{\mathbf{k}}\right|^{2}+\left|b_{\mathbf{k}}\right|^{2}\right) d \mathbf{k}=1$.

The relation (24) shows that resonance frequency depends upon the average domain size $d$. For macroscopic domains with $d>>\lambda \equiv s /\left(g H_{s f}\right)$ characteristic value of $k \sim 1 / d<<s / \omega$ and the last term in (24) can be neglected. In this case the domain can be treated as infinite, corresponding AFMR frequency is

$$
\Omega_{A F M R}^{(\infty)}=g \sqrt{H_{s f}^{2}+H_{M E}^{2}}
$$

and we arrive to a standard situation with the «frozen» lattice, AFMR gap is defined by anisotropy and magnetoelasticity as well. In the opposite case with $d<<\lambda$, the Fourrier spectrum of functions $\sin 2 \varphi(\mathbf{r})$ and $\cos 2 \varphi(\mathbf{r})$ has two significant contributions with $k=0$ and $k=\pi / d$. The corresponding expression for AFMR frequency is

$$
\begin{gathered}
\Omega_{A F M R}=g\left(H_{s f}^{2}+\frac{H_{0}^{4} H_{M E}^{2}}{4 H_{M D}^{4}}\right)^{1 / 2} \times \\
\times\left[1+\frac{g^{2} H_{M E}^{2} d^{2}}{2 \pi^{2} s^{2}}\left(\left|a_{\pi / d}\right|^{2}+\left|b_{\pi / d}\right|^{2}\right)\right]<\Omega_{A F M R}^{(\infty)},
\end{gathered}
$$

where we have taken into account that

$$
\left|a_{0}\right|^{2}+\left|b_{0}\right|^{2}=\langle\cos 2 \varphi\rangle^{2}+\langle\sin 2 \varphi\rangle^{2}=\frac{H_{0}^{4}}{4 H_{M D}^{4}} .
$$

So, in the magnetically inhomogeneous sample the crystal lattice does follow the oscillations of the magnetic moments and thus diminishes the magnetoelastic contribution to the resonance fre-

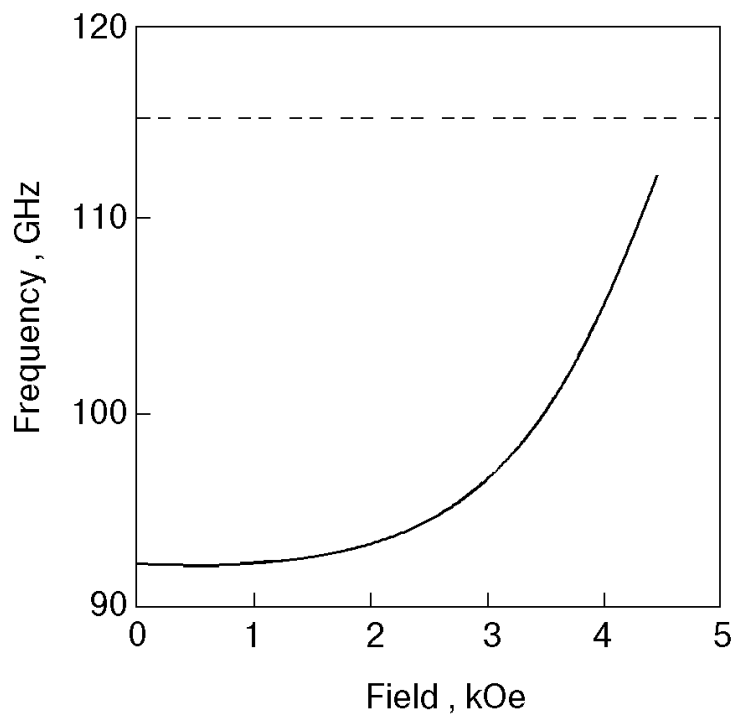

Fig. 4. Theoretical field dependence of resonance AFMR frequency (scheme): solid line - polydomain sample; dash line infinite homogeneous sample.

quency [see formula (26)]. This effect should be more pronounced in $\mathrm{CoCl}_{2}$, where magnetoelastic contribution into AFMR spectrum is of the same order as an anisotropy one.

The field dependence of AFMR spectrum in $\mathrm{CoCl}_{2}$ can be thus explained as follows. Suppose, at zero field the sample has a well developed DS with the average size $d \leq \lambda$ ( for $\mathrm{CoCl}_{2} \lambda \sim 10^{-7} \mathrm{~m}$ ). The lattice is then proves to be partially «unfrozen» and frequency is defined mainly by anisotropy field [see expression (26)]. The external magnetic field affects the AFMR frequency in two ways: through the variation of average cosine and sine values and through the increase of the average domain size ${ }^{*}$ $d\left(H_{0}\right)$ [last term in (26)]. As a result, the frequency grows smoothly with the field $H_{0}$. After the domain size achieves macroscopic value $d \sim R>>\lambda$, the lattice becomes frozen, and resonance frequency steeply jumps to the value corresponding to infinite homogeneous sample [formula (25)]. Tentative behavior of AFMR vs magnetic field calculated from (25), (26) with $g=6, H_{s f}=2 \mathrm{kOe}, H_{M E}=1.5 \mathrm{kOe}$, $H_{M D}=3.3 \mathrm{kOe}$ is shown in Fig. 4.

Additional decrease of AFMR frequency in the polydomain sample can also result from the damping of the domain wall motion, stimulated by reassembling of the DS in the external magnetic field [16]. The ultimate value of AFMR frequency, $\tilde{\Omega}_{A F M R}=\sqrt{\Omega_{A F M R}^{2}-\delta^{2}}$, where $\delta$ is the damping coefficient, can be significantly lower than $\Omega_{A F M R}^{(\infty)}$.

* We suppose that the change of the DS proceeds by the growth of the domains of certain type at the expence of others. 
It can be shown that the model of immobile domain walls gives another form of spectrum which does not correlate with the experimental data.

\section{Discussion}

Above we propose the model which naturally interprets the antiferromagnetic domains in the easy-plane antiferromagnets as equilibrium. The model is based on the magnetoelastic origin of the domains, supposition of mobile domain walls and additional condition imposed on the average strain of the sample. For illustration we have considered the simplest example when the condition in question was related with the surface tension of the sample which was the case if the surface energy made a significant contribution into free energy of the sample. As a consequence of the model, the value of monodomenization field depends upon the characteristic size $R$ of the sample and thus vanishes when $R$ tends to infinity.

The results obtained could be generalized if $R$ implies the characteristic size of substructure unit of the sample (i.e., dislocation-free region or crystallite), defined by the technological factors. In the case of fixed (glued) sample expression (2) should be substituted with corresponding boundary conditions for the sample faces. Anyway, finite-size effects can play a crucial role in the behavior of antiferromagnets with significant magnetostriction and should be taken into account in interpretation of experimental results.

The predicted behavior of the DS of AFM is analogous to that of ferromagnetic. Namely, up to some critical external field value, the effective internal magnetic field is compensated due to the reassembling of the domains. The value of critical field is defined by the geometry and size of the sample. It is interesting and important that in AFM model considered the long range interactions include no magnetic component and are completely provided by elasticity.

The size of the domains can be calculated with the account of short-wavelength contribution to the surface energy which compensates the increase of volume energy resulting from the domain walls.

In our calculations we have not considered the magnetostatic effects and domain walls themselves. Demagnetization factor influences the DS in the region of 1-st order (spin-flop) phase transition which takes place in narrow interval of angle $\psi \approx 0$ (see [4]). For arbitrary orientation of the external magnetic field in the easy plane of AFM the magnetostatic contribution is $\left(H_{M D} / 2 H_{E}\right) \sim$ $\sim 0.06<<1$ times smaller than the surface energy and thus can be neglected.
The account of domain walls is necessary for evaluation of the domain size that is out of scope of the present paper. The structure of domain walls can be calculated in an analogy with general approaches (see [3]) by taking into account magnetic and elastic subsystems. It should be noted that in the defect-free sample the interdomain boundary are ideally conjugated and no stresses appear because of compatibility conditions.

\section{Conclusions}

1. The domain structure of easy-plane AFM with degenerated orientation of antiferromagnetic vector can be treated as equilibrium in the finite-size sample with the mobile domain walls. The effect originates from the magnetoelastic nature of the domains with the account of surface tension.

2. At zero magnetic field all types of the domains are equally represented. The external magnetic field effects the magnetic and elastic properties of the sample in two ways. At small field value variation of the domain structure gives rise to additional average strain field which compensates the external magnetic field, the orientation of the magnetic moments inside domains being fixed. At some critical value, $H_{0}=H_{c}$, the sample becomes monodomain and magnetic field results in reorientation of the magnetic moments.

3. Experimentally observed magnetostriction vs magnetic field dependence for $\mathrm{CoCl}_{2}$ crystal is adequately described in terms of the model under consideration with mobile domain walls.

4. AFMR frequency of polydomain crystal can be significantly lowered due to «unfreezing» of the crystal lattice if the size of domain is quite small.

5. The above mentioned calculations can be considered as a basis for the following important and quite general supposition: variation of the surface energy (that is of Coulomb nature and so is not small) can result in the formation of equilibrium inhomogeneous state (or in other words, the equilibrium domain structure of magnetoelastic or elastic nature).

\section{Acknowledgements}

We are sincerely indebted to Prof. S. M. Ryabchenko, Dr. A. F. Lozenko and Dr. V. M. Kalita for stimulating and constructive discussions of the problem under consideration and for opportunity to get acquainted with the results of the manuscript [5] before publication. We are also grateful to Prof. V. G. Bar'yakhtar for the interest to the work and useful comments. 
1. M. M. Fartdzinov, Usp. Fiz. Nauk 84, 611 (1964) [Sov. Phys. Usp. 7, 853 (1964)].

2. V. V. Eremenko and N. F. Kharchenko, Sov. Sci. Review, Sec. A5, 1 (1984)

3. V. G. Bar'yakhtar, A. N. Bogdanov, and D. A. Yablonskii, Usp. Fiz. Nauk 156, 47 (1988) [Sov. Phys. Usp. 31, 810 (1988)].

4. A. N. Bogdanov and I. E. Dragunov, Fiz. Nizk. Temp. 24, 1136 (1998) [Low Temp. Phys. 24, 852 (1998)].

5. V. M. Kalita, A. F. Losenko, S. M. Ryabchenko, and P. A. Trotsenko, Ukr. Fiz. Zh. 43, 1469 (1998).

6. K. Wilkinson, J. W. Cable, E. O. Wollan, and W. C. Koehler, Phys. Rev. 113, 497 (1959).

7. A. F. Lozenko, P. E. Parkhomchuk, S. M. Ryabchenko, and P. A. Trotsenko, Fiz. Niz. Temp. 14, 941 (1988) [Sov. J. Low Temp. Phys. 14, 317 (1988)].

8. K. Otsuka, C. M. Wayman, K. Nakai, H. Sakamoto, and K. Shimizu, Acta Met. 24, 207 (1976).
9. E. A. Turov, Physical Properties of Magnetically Ordered Crystals, Ed. AN SSSR, Moscow (1963).

10. S. V. Maleev, Pis'ma Zh. Eksp. Theor. Fiz. 68, 67 (1998).

11. A. S. Borovik-Romanov and E. G. Rudashevskii, Zh. Eksp. Theor. Fiz. 47, 2095 (1964) [Sov. Phys. JETP 20, 1407 (1965)].

12. E. A. Turov and V. G. Shavrov, Fiz. Tverd. Tela 7, 217 (1965).

13. A. V. Andrienko and L. A. Prozorova, Zh. Eksp. Theor. Fiz. 74, 1527 (1978) [Sov. Phys. JETP 47, 798 (1978)].

14. V. V. Gann and A. I. Zhukov, Fiz. Tverd. Tela 24, 2799 (1982).

15. B. A. Ivanov and G. K. Oksyuk, Nonlinear Dynamics of the Weak Antiferromagnets, Preprint ITPh, ITF-84-8P, Kiev (1984).

16. V. A. Loktev and Yu. B. Gaididei, Fiz. Nizk. Temp. 9, 204 (1983) [Sov. J. Low Temp. Phys. 9, 104 (1983)]. 